\title{
Phase Transitions in Staggered Spin Ladders
}

\author{
M. A. Martín-Delgado, ${ }^{1}$ R. Shankar, ${ }^{2}$ and G. Sierra ${ }^{3}$ \\ ${ }^{1}$ Departamento de Física Teórica I, Universidad Complutense, 28040 Madrid, Spain \\ ${ }^{2}$ Sloane Physics Laboratories, Yale University, New Haven, Connecticut 06520 \\ ${ }^{3}$ Instituto de Matemáticas y Física Fundamental, C.S.I.C. Serrano 123, 28006 Madrid, Spain
}

(Received 8 May 1996)

\begin{abstract}
We map spin ladders with $n_{l}$ legs and couplings $J^{\prime}$ across all rungs and $J(1 \pm \gamma)$ along the legs, staggered in both directions, to a sigma model. Setting its $\theta=(2 m+1) \pi$ (where it is known to be gapless), we locate the critical curves in the $\gamma$ versus $J^{\prime} / J$ plane at each $n_{l}$, and spin $S$. The phase diagram is rich and has some surprises: When two gapped chains are suitably coupled, the combination becomes gapless. With $n_{l}, \gamma$, and $J^{\prime} / J$ to control, the prospects for experimentally observing any one of these equivalent transitions are enhanced. We interpret our results in the framework of the resonating valence bond description of ladders. [S0031-9007(96)01410-X]
\end{abstract}

PACS numbers: 75.10.Jm, 75.30.Ds, 75.50.Ee

With this paper we contribute to the explosive growth in the theoretical and experimental studies of antiferromagnetic spin chains and ladders. Recall Haldane's [1] mapping of the spin- $S$ Heisenberg chain with Hamiltonian

$$
H=J \sum_{n} \mathbf{S}(n) \cdot \mathbf{S}(n+1)
$$

to the nonlinear sigma model with euclidean action

$S=\int d x d \tau\left[-\frac{1}{2 g}(\nabla \boldsymbol{\Phi})^{2}+i \frac{\theta}{4 \pi} \boldsymbol{\Phi} \cdot \partial_{x} \boldsymbol{\Phi} \times \partial_{\tau} \boldsymbol{\Phi}\right]$.

Here $\Phi$ is a unit three vector, and $\theta$, which multiplies $i$ times the integer valued winding number $W$, is

$$
\theta=2 \pi S \text {. }
$$

An alternate derivation was given by Affleck [2].

Since $\theta$ enters via $e^{i \theta W}$, (i) it matters only $\bmod 2 \pi$, (ii) when $\theta=0, \pi$ the path integral is invariant under $x \rightarrow-x$ (parity) under which $W \rightarrow-W$, and (iii) all integer (half-integer) spin chains have essentially $\theta=0$ $(\pi)$. The $\theta=0$ model is known to have exponentially decaying correlations [3]. As for $\theta=\pi$, since the spin$\frac{1}{2}$ Bethe chain is gapless, so must be the $\theta=\pi$ sigma model, provided the mapping to the sigma model (most reliable for large $S$ ) is valid down to spin- $\frac{1}{2}$. In addition, Shankar and Read [4] have shown independently that the sigma model at $\theta=\pi$ is massless by considering the $\tau$ continuum Hamiltonian of the lattice regulated model. It is also accepted that nonstaggered ladders with $n_{l}$ legs are gapless only if $n_{l} S$ is half-integer [5-9]. This is most transparent when the interleg coupling $J^{\prime}$ is much larger than the intraleg coupling $J$, for we can first solve the problem of $n_{l}$ spins along a rung, take the lowest energy multiplet in each rung, and then couple them with $J$, thereby getting a single chain, about which everything is known. One then verifies that nothing changes as $J^{\prime}$ is lowered. Equivalently [10,11], one can show that the topological terms for the chains are additive, giving $\theta=2 \pi n_{l} S$. Different modifications of spin ladders can also be considered by adding next-to-nearest neighbor couplings $[12,13]$. Coupled spin chains have been studied by combining mean field theory techniques with exact results for one chain [14].

The spin systems to be considered here have a very important feature: They have staggered weights. Let us then begin with a single chain for which

$$
J(n)=J\left(1+(-1)^{n} \gamma\right)
$$

is the coupling between sites $n$ and $n+1$. Notice that $\gamma \rightarrow-\gamma$ amounts to sublattice exchange $n \rightarrow n+1$ and that the restriction $|\gamma|<1$ keeps the interaction antiferromagnetic. Affleck and Haldane [2,15] showed that, in this case,

$$
\theta=2 \pi S(1+\gamma)
$$

so that when $\gamma$ is varied from -1 to $+1, \theta$ passes an odd multiple of $\pi$, i.e., the system is critical exactly $2 S$ times. It is instructive to interpret these transitions in the valence bond terminology of Affleck et al. (AKLT) [16], wherein each spin- $S$ is viewed as a symmetrized product of $2 S$ spinors. As $\gamma$ is raised from -1 , the chain goes from being fully dimerized with all the valence bonds (spinor contractions) on odd- $n$ links to being dimerized with all valence bonds on the even- $n$ links. As each spinor index switches loyalty, it necessarily reaches a point when it can equally well go either way, producing a nonstaggered, i.e., a gapless spin- $\frac{1}{2}$ chain. (The effective interaction of these spin- $\frac{1}{2}$ degrees of freedom can be nonstaggered even though the original Heisenberg interaction is.)

We are interested here in staggered chains shown in Fig. 1, with antiferromagnetic horizontal couplings on the $a$ th leg $\left(a=1, \ldots, n_{l}\right)$ obeying

$$
J_{a}(n)=J\left(1+(-1)^{n+a+1} \gamma\right),
$$

i.e., staggered in both directions. This is a novel case not considered before, and it is by far the most interesting. We 


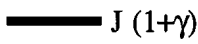

$\mathrm{J}(1-\gamma)$

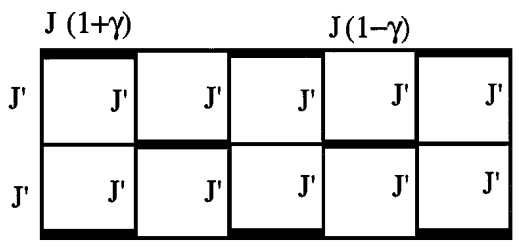

FIG. 1. A ladder with staggered couplings $J(1 \pm \gamma)$ along the horizontal legs and $J^{\prime}$ along the vertical rungs.

now show that such systems have a rich phase structure in the $\gamma$ versus $J^{\prime} / J$ plane at each $n_{l}$ and $S$. We expect that these systems should also be experimentally realizable. Another family, with alternation in the leg direction only $\left[J_{a}(n)=J\left(1+(-1)^{n} \gamma\right)\right]$, is interesting only if $J^{\prime}$ is ferromagnetic, and will be briefly discussed later.

Let us begin with Affleck's derivation of the sigma model Hamiltonian from the spin chain by pairing spins, forming their difference and sum, and turning these into the sigma model field and its conjugate momentum, respectively, in the limit of large $S$. This method was generalized by Sierra [11] to uniform ladders. The main difference was the $n_{l}$-fold increase in the number of degrees of freedom due to the transverse label $a=$ $1, \ldots, n_{l}$ for the legs. A low energy analysis indicated that only one of these modes remained low in energy and defined the effective sigma model, while the rest vanished as $J^{\prime}$ goes to zero. This effective model had $\theta=2 \pi n_{l} S$ (independent of couplings), yielding the previously quoted result for nonstaggered ladders, namely, that only an odd number of half-integer chains were massless. We have been able to extend this derivation to the staggered ladders and found

$$
\theta=2 \pi S n_{l}\left(1+\gamma f_{n_{l}}\left(J^{\prime} / J\right)\right)
$$

where

$$
\begin{aligned}
f_{n_{l}}\left(J^{\prime} / J\right)=\frac{1}{n_{l}^{2}}[ & \delta_{n_{l}, \text { odd }} \\
& +2 \sum_{m=1,3, \ldots, n_{l}-1} \frac{1}{\sin ^{2}\left(\frac{\pi m}{2 n_{l}}\right)} \\
& \left.\times \frac{1}{1+\frac{J^{\prime}}{J} \cos ^{2}\left(\frac{\pi m}{2 n_{l}}\right)}\right],
\end{aligned}
$$

with $\delta_{n_{l} \text {,odd }}$ equal to 1 if $n_{l}$ is odd and zero otherwise. We refer the reader to Sierra [11] for a very similar derivation in the uniform case.

The critical points follow from setting this $\theta$ equal to odd multiples of $\pi$. We explore two cases now: $n_{l}=2, S=\frac{1}{2}, 1$, which should convince the reader of the soundness of this method, and facilitate the discussion of the cases with larger $S$ and $n_{l}$.
Consider Fig. 2(a). On the $\gamma$ axis, where the chains decouple, there is just one critical point corresponding to the nonstaggered spin- $\frac{1}{2}$ chain. We predict that, as $J^{\prime}$ grows, this becomes two critical points that move toward the walls $|\gamma|=1$. Although staggering or interchain coupling are individually bad for criticality, a certain combination seems to restore criticality. Can we believe this? Consider $\gamma=-1$. Each chain breaks up into disconnected pairs, but the disconnected pairs of one chain do not lie opposite to those of the other, but are displaced by one unit. When these get coupled by $J^{\prime}$, we have a "snake" chain that winds through the lattice. It is a spin- $\frac{1}{2}$ chain with alternating weights $2 J$ and $J^{\prime}$. Clearly, $J^{\prime}=2 J$, and becomes critical as predicted by the theory. Thus the vertical $J^{\prime}$ axis is seen to play the role of an effective $\gamma$ for the snake. We display this by showing three snakes in the left margin of Fig. 2, with vertical bonds which are stronger than, equal to, and weaker than the horizontal ones $(2 J)$. The same thing happens on $\gamma=+1$, with $n \rightarrow n+1$. It is not as easy to understand criticality as we go into the rectangle, but, by continuity of $\theta$, the critical curve must exist. There is, however, one caveat: The phase diagram in Fig. 2 does not strictly follow from the equation for $\theta$ when $J^{\prime} \rightarrow 0$; the two critical curves coming down from $J^{\prime} / J=2$ on $|\gamma|=1$ will cut the $\gamma$ axis at distinct points on either side of the origin instead of meeting there. But we know that the sigma model mapping is doomed to fail as $J^{\prime} \rightarrow 0$ : We will not get one low energy field (the putative sigma model field) but two, since the gap that separated the sigma model field from the other vanishes with $J^{\prime}$. Fortunately, on the $\gamma$ axis, where the chains decouple, we know everything:

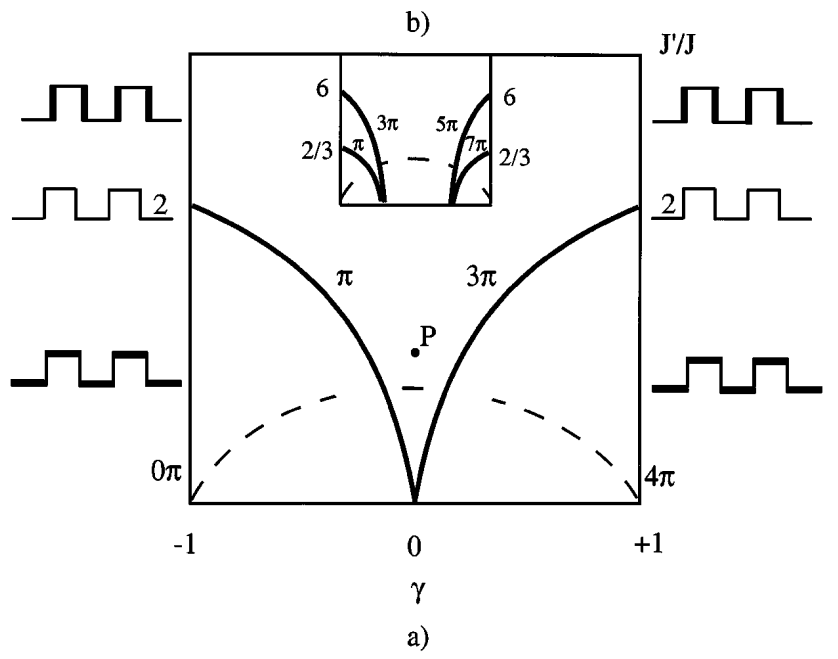

FIG. 2. (a) Phase diagram for staggered ladders with $n_{l}=2$ and spin- $\frac{1}{2}$. The solid lines represent critical lines corresponding to the $\theta$ parameter being an odd multiple of $\pi$ (the dashed line is just indicative for counting the number of critical lines between $\gamma=-1$ and $\gamma=+1)$. We also show the bonds associated with critical (nonstaggered) and gapped snakes (staggered). (b) The inset shows the same as above, but with spin-1. 
There is only one transition at $\gamma=0$ which the two chains undergo simultaneously. The final phase diagram is the union of what we know on the $\gamma$ axis (about decoupled chains) with what we know off the $\gamma$ axis (from the sigma model) [17]. Even off the $\gamma$ axis the sigma model is only to be taken as a guide to the topology of the phase diagram and not for the exact location of the critical curves. This is because the formula for $\theta$ is generally not exact except when $\gamma=0$ and $\theta=2 \pi n_{l} S$, in which case the sigma model is invariant under parity and you cannot alter $\theta$ by a small amount (say, of order $1 / S$ ) without violating parity. Along an arc [shown by a dotted line in Fig. 2(a)] starting at $\gamma=-1, J^{\prime}=0$ and ending at $\gamma=1, J^{\prime}=0, \theta$ rises continuously from 0 to $4 \pi$. The critical behavior is the same across any of these critical curves and the gap will behave as $t^{2 / 3}$, where $t$ is the control parameter, as predicted by Cross and Fisher [18]. (There will be logarithmic corrections since the $\theta=\pi$ sigma model differs from the conformally invariant WessZumino-Witten model by a marginally irrelevant operator [4]. Chitra et al. [13] avoid the $\log _{1}$ by adding a special value of nnn coupling to the spin- $\frac{1}{2}$ chain and find an exponent very close to $2 / 3$.)

Let us examine Fig. 2(a) in terms of the resonating valence bond picture of White, Noack, and Scalapino [7] for a nonstaggered $n_{l}=2, S=\frac{1}{2}$ system, taking a typical point $P$ vertically above the origin in Fig. 2(a). In the absence of defects, the bonds in each $2 \times 2$ square resonate between being vertical (with coupling $J^{\prime}$ ) and horizontal (with coupling $J$ ). A defect forces the bonds to be horizontal, staggered, and nonresonating until we reach the next defect. This causes a linear confining potential and restricts the excitations to spin-1. In our problem, we are free to move off this point towards negative $\gamma$. Now the staggered horizontal bond configuration between the defects becomes more favorable, and we soon hit the critical curve on which the staggered bonds' configuration becomes degenerate with the resonant ones, and the defects (spinons) are liberated. To the left of the critical curve, confinement resumes, since we now reach a phase where staggered bonds are favored over resonant ones. If we drop vertically from the critical point to the $J^{\prime}=0$ axis, we have decoupled staggered chains. The bonds are dimerized in the preferred sublattices. A pair of defects now forces singlets on unfavorable bonds in the region in between. When $J^{\prime}$ is turned on, the bonds can resonate between the defects, since the defect has lined them up across each other. Increasing $J^{\prime}$ improves resonance, and we finally hit the critical curve. All critical curves may be characterized as those on which the defects are deconfined. As for the order parameter for the different phases, it is best to move up the $\gamma=-1$ axis, where we see that the valence bonds go from being horizontal to vertical. This is just the Affleck-Haldane transfer of bonds on a chain, but along the length of the snake, where even/odd bonds turn into vertical/horizontal bonds.
Consider Fig. 2(b) for spin-1. Once again, on $\gamma=-1$, we get a spin-1 snake, which becomes gapless when its staggering equals $\pm 1 / 2$ according to the sigma model (5) [19]. The ratio of couplings $J^{\prime} / 2 J$ determines the effective staggering along the snake. Setting $J^{\prime} / 2 J$ equal to $(1 \pm$ $\left.\frac{1}{2}\right) /\left(1 \mp \frac{1}{2}\right)$, we get critical values $J^{\prime} / 2 J=3,1 / 3$. We can adapt the nonlocal order parameter of den Nijs and Rommelse [20] (rendered along the snake) to describe the $Z_{2}$ symmetries.

Now for larger values of $S$ and $n_{l}$. Each singlechain transition on the $\gamma$ axis splits into $n_{l}$ transitions as we turn on $J^{\prime}$. The critical curves bend towards the wall $(|\gamma|=1)$ nearest to them. The parameter $\theta$ rises continuously from 0 to $4 \pi n_{l} S$ as we follow the arc shown in Fig. 2(a). There are, however, some differences. First, we get honeycomb ladders instead of snakes for larger $n_{l}$. Next, we no longer have an easy way to see that the sigma model is even qualitatively correct when it locates critical lines for us. However, we expect the model to be weakest when $n_{l}$ or $S$ is small. Having passed the test there, it seems immune to further jeopardy. Finally, if $n_{l} S$ is half-integer, an odd number of lines will emanate from the origin, one of which will go straight up to $J^{\prime}=\infty$ (corresponding to nonstaggered odd- $n_{l}$ half-integer spin chain ladders, known to be gapless). The top half of Fig. 3 illustrates this for the case $S=1 / 2, n_{l}=3$.

The phase diagrams have a natural extension to $J^{\prime}<$ 0 , provided $J_{a}(n)=J\left(1+\gamma(-1)^{n}\right)$, i.e., the staggering is only along the leg but not along the rung direction (columnar staggering [21]). Now, if we lower $J^{\prime}$ from 0 to $-\infty$, each transition point of the decoupled spin- $S$ chain splits into $n_{l}$ lines, and all $2 n_{l} S$ of them flow down to $J^{\prime}=-\infty$ and terminate at the $\gamma^{\prime}$ 's corresponding to the $2 n_{l} S$ transitions of the spin $n_{l} S$ chain. The bottom half of Fig. 3 shows this for $n_{l}=3, S=1 / 2$. The similarity between these diagrams and those of coupled quantum Hall layers will be discussed elsewhere.

To summarize, we have considered the phase diagram of ladders with staggered couplings by mapping to a sigma

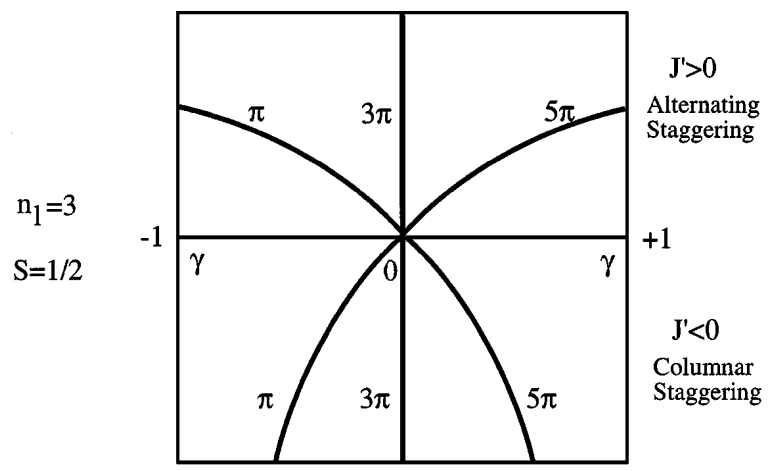

FIG. 3. Top half shows the phase diagram for staggered ladders with $n_{l}=3$ and spin- $\frac{1}{2}$ with staggering in both directions and $J^{\prime}>0$, while the bottom describes $J^{\prime}<0$ and staggering only along the leg direction. 
model and setting its $\theta$ to an odd multiple of $\pi$. There were a few surprises: We have examples here wherein coupling gapped chains leads to gapless chains because staggering and interchain couplings, which separately destroy gaplessness, can conspire to keep the system gapless. Thus two spin- $\frac{1}{2}$ chains with small staggering and small $J^{\prime}$ can remain massless. At all these phase transitions, the gap will vanish as $t^{2 / 3}$ (up to logarithms). We anticipate confirmation of our predictions by Monte Carlo, density matrix renormalization group, series expansions, etc. The sigma model complements these approaches: It does not do very well numerically, but manages to give at one stroke the phase diagram for any choice of $S$ and $n_{l}$. For instance, we know that, on $\gamma= \pm 1$, where we have a honeycomb ladder, each transition of a single chain gets transformed into $n_{l}$ transitions as $J^{\prime}$ is varied. This accumulation of critical points (for any spin, half-integer or otherwise) facilitates extrapolation to the ordered state in $d=2$, although we cannot raise $n_{l}$ too much.

We urge experimentalists [22] to find ladders wherein bonds alternate in one or both directions (6), and either $\gamma$ or $J^{\prime} / J$, or both, can be varied at least slightly; or to find the honeycomb ladders, an extreme case of bond alternation $(\gamma= \pm 1)$. Once any such a ladder is found, it will have many transitions, whatever be the spin; a ladder with four legs and spin 1 will have four transitions as $J^{\prime}$ varied, say, by applying pressure.

R.S. thanks I. Affleck, E. Dagotto, M.P.A. Fisher, H. Fukuyama, L. P. Regnault, and S. Sachdev for useful conversations, the Instituto de Matemáticas y Física Fundamental (C.S.I.C), and the Institute of Theoretical Physics at Santa Barbara. This work is partly supported by CICYT under Contract No. AEN93-0776 (M.A.M.D.), NSF Grants No. DMR 9120525 and No. PHY9407194 (R.S.), and by the Spanish Fund PB92-1092 and European Community Grant No. ERBCHRXCT920069 (G. S.).

[1] F. D. M. Haldane, Phys. Rev. Lett. 50, 1153 (1983); Phys. Lett. 93A, 464 (1983).

[2] I. Affleck, in Fields, Strings, and Critical Phenomena, Les Houches Lecture Notes, edited by E. Brezin and J. ZinnJustin (North-Holland, Amsterdam, 1988).

[3] C. J. Hamer, J. B. Kogut, and L. Susskind, Phys. Rev. D 19, 3091 (1979); A. Polyakov and P. B. Weigman, Phys. Lett. 131B, 121 (1983).
[4] R. Shankar and N. Read, Nucl. Phys. B336, 457 (1990).

[5] E. Dagotto, J. Riera, and D. Scalapino, Phys. Rev. B 45, 5744 (1992).

[6] T. Barnes, E. Dagotto, J. Riera, and E. Swanson, Phys. Rev. B 47, 3196 (1993).

[7] S. White, R. Noack, and D. Scalapino, Phys. Rev. Lett. 73, 886 (1994).

[8] S. Gopalan, T. M. Rice, and M. Sigrist, Phys. Rev. B 49, 8901 (1994).

[9] E. Dagotto and T. M. Rice, Science 271, 618 (1996).

[10] D. V. Khveshchenko, Phys. Rev. B 50, 380 (1994); For a two leg ladder, see D. Sénéchal, Report No. cond-mat 9506026 (to be published).

[11] G. Sierra, J. Phys. A 29, 3299 (1996).

[12] S. R. White, Phys. Rev. B 53, 52 (1996).

[13] R. Chitra, S. Pati, H. R. Krishnamurti, D. Sen, and S. Ramasesha, Phys. Rev. B 52, 6581 (1995); S. Brehmer, H. J. Mikeska, and U. Neugebauer, Report No. condmat/9604136 (to be published).

[14] H. J. Schulz, Report No. cond-mat/9604144 (to be published); Phys. Rev. B 34, 6372 (1986).

[15] I. Affleck and F.D. Haldane, Phys. Rev. B 36, 5291 (1987).

[16] I. Affleck, T. Kennedy, E. H. Lieb, and H. Tasaki, Commun. Math. Phys. 115, 477 (1988).

[17] Our picture is also supported by D. G. Shelton, A. A. Nersesyan, and A.M. Tsvelik, Phys. Rev. B 53, 8521 (1996); J. Oitmaa, R. R.P. Singh, and Z. Weihong, Report No. cond-mat/9602055 (to be published); S.P. Strong and A.J. Millis, Phys. Rev. Lett. 69, 2419 (1992).

[18] M. C. Cross and D. S. Fisher, Phys. Rev. B 19, 402 (1979).

[19] From Quantum MC and DMRG studies, the precise position of these points is \pm 0.25 as in S. Yamamoto, J. Phys. Soc. Jpn. 63, 4327 (1994); Y. Kato and A. Tanaka, J. Phys. Soc. Jpn. 63, 1277 (1994). Similar studies for spin-2 can be found in M. Yamanaka, M. Oshikawa, and S. Miyashita, Report No. cond-mat/9604107, 1996 (to be published).

[20] M. den Nijs and K. Rommelse, Phys. Rev. B 40, 4709 (1989); D. Arovas and S. Girvin, Bull. Am. Phys. Soc. 33, 797 (1988); H. Tasaki, Phys. Rev. Lett. 66, 798 (1991); M. Kohomoto and H. Tasaki, Phys. Rev. B 46, 3486 (1992).

[21] K. Totsuka and M. Suzuki, J. Phys. Condens. Matter 7, 6079 (1995); (to be published).

[22] See, for example, M. Azuma, Z. Hiroi, M. Takano, K. Ishida, and Y. Kitaoka, Phys. Rev. Lett. 73, 3463 (1994); D. C. Johnston, J. W. Johnson, D. P. Geshorn, and A. J. Jacobson, Phys. Rev. B 35, 219 (1987). 